\title{
Prediction of gestational age based on genome-wide differentially methylated regions
}

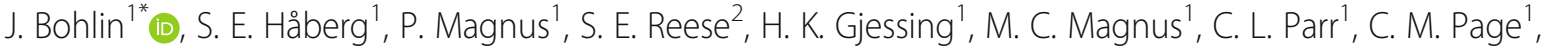 \\ S. J. London ${ }^{2+}$ and W. Nystad ${ }^{1+}$
}

\begin{abstract}
Background: We explored the association between gestational age and cord blood DNA methylation at birth and whether DNA methylation could be effective in predicting gestational age due to limitations with the presently used methods. We used data from the Norwegian Mother and Child Birth Cohort study (MoBa) with Illumina HumanMethylation450 data measured for 1753 newborns in two batches: MoBa 1, n= 1068; and MoBa 2, n=685. Gestational age was computed using both ultrasound and the last menstrual period. We evaluated associations between DNA methylation and gestational age and developed a statistical model for predicting gestational age using MoBa 1 for training and MoBa 2 for predictions. The prediction model was additionally used to compare ultrasound and last menstrual period-based gestational age predictions. Furthermore, both CpGs and associated genes detected in the training models were compared to those detected in a published prediction model for chronological age.
\end{abstract}

Results: There were $5474 \mathrm{CpGs}$ associated with ultrasound gestational age after adjustment for a set of covariates, including estimated cell type proportions, and Bonferroni-correction for multiple testing. Our model predicted ultrasound gestational age more accurately than it predicted last menstrual period gestational age.

Conclusions: DNA methylation at birth appears to be a good predictor of gestational age. Ultrasound gestational age is more strongly associated with methylation than last menstrual period gestational age. The CpGs linked with our gestational age prediction model, and their associated genes, differed substantially from the corresponding CpGs and genes associated with a chronological age prediction model.

\section{Background}

Determination of gestational age (GA) is important for assessing due dates, giving adequate prenatal care, and suggesting appropriate interventions in preterm and post-term pregnancies. In Norway it is common clinical practice to assign pregnant women a due date based on the date of the last menstrual period (LMP). Around pregnancy week 18 a routine ultrasound examination, attended by almost all pregnant women, is used to calculate more precise estimates of GA and due date [1]. Although ultrasound-based estimates of GA are more precise in predicting the birth date than estimates based

\footnotetext{
* Correspondence: jon.bohlin@fhi.no

${ }^{\dagger}$ Equal contributors

${ }^{1}$ Norwegian Institute of Public Health, P.O. Box 44040456 Oslo, Norway

Full list of author information is available at the end of the article
}

on the last menstrual period, LMP-based estimates may be preferred in some circumstances $[2,3]$. There is wide variability in estimated GA even when ultrasound is used [4]. Ultrasound GA predictions eliminate uncertainties such as inaccurate reporting of the date of LMP and variability in the follicular phase length, although they assume uniform fetal growth during early pregnancy, which is only approximately true [5]. Hence, there is room for more accurate methods for estimating GA, which would be of great benefit in clinical practice.

There is growing interest in understanding the role of DNA methylation and its relation to GA in newborns. A study comprising 259 newborns, based on the Illumina HumanMethylation27 platform mapping approximately 28,000 CpG sites genome-wide and covering close to half the genes in the human genome, identified a number of 
candidate genes that were associated with GA [6]. Employing the CHARM 2.0 assay consisting of 2.1 million probes (covering 5.2 million probes arranged into probe groups) [7], Lee et al. performed a similar genome-wide screening, from a sample size of 187 newborns, and identified differentially methylated CpGs in the neighborhood of three genes (NFIX, RAPGEF2, and MSRB3) [8]. A more recent study, based on the Illumina HumanMethylation450 technology and longitudinal data from the Avon Longitudinal Study of Parents and Children (ALSPAC) cohort [9], found an association between DNA methylation and GA that appears to fade by early childhood [10]. Finally, birth weight correlates with GA and it has been shown that DNA methylation is associated with birth weight in newborns [11].

There has also been increased interest in exploring the relationship between DNA methylation and chronological age in humans. One study reported a fairly precise prediction of chronological age in humans using a small set of CpGs identified from several DNA methylation datasets [12]. These datasets were generated from many different studies, all of which were based on the Illumina HumanMethylation450 platform, assessing epigenetic effects from a multitude of cell types and disease-specific endpoints [12]. The strong relationship between DNA methylation and chronological age indicates that methylation analysis may be used as a tool in forensic age prediction [13]. Given the previous studies showing robust methylation signatures of GA, we reasoned that methylation data could also be used to predict GA.

The Norwegian Mother and Child Cohort Study (MoBa) $[14,15]$ contains genome-wide methylomes, as mapped by the Illumina HumanMethylation450 platform, for 1753 newborns. The 1753 newborns were sampled from the MoBa cohort and the methylation data were generated at two different points in time. The first batch, henceforth referred to as MoBa 1, containing 1068 newborns, was extracted and processed in 2011. The second batch, MoBa 2 , which in the present study is only used for replication, includes 685 newborns and was processed in 2013. MoBa also had information on GA at birth estimated from both ultrasound measurements, near pregnancy week 18 , as well as the LMP. Having a larger sample size than previous studies, we wanted to further explore the associations between GA and DNA methylation and the possibility of estimating GA, as has been demonstrated for chronological age [12]. We also examined whether the CpGs and genes that predict chronological age overlapped with CpGs and genes we found predictive for GA.

\section{Results and discussion}

\section{Gestational age and methylation}

Robust MM-type linear regression was performed on MoBa 1 with CpG sites ( $\beta$ values) as the outcome variables.
After correcting for a set of covariates (see the "Methods" section and Table 1; Table 2 contains the corresponding covariate statistics for the MoBa 2 dataset), we found approximately 5474 differentially methylated $\mathrm{CpGs}$ associated with GA calculated using ultrasound (10,784 CpGs when LMP-based estimations were used) after adjusting for multiple testing using Bonferroni correction $\left(p_{B}<0.05,473,731\right.$ tests; Fig. 1). Figure 2 indicates that the significant $\mathrm{CpGs}$ were predominantly decreased in methylation (3911 versus 1563), which may suggest that increasing GA is associated with increased expression of genes linked to the differentially methylated probes as the CpGs mapped by the Illumina HumanMethylation450 platform are predominantly located in the promotor regions [16]. Using the less strict false discovery rate (FDR) correction for multiple testing [17], 44,359 probes $(44,544$ probes for LMP estimations) were found to be significant $(\mathrm{q}<0.05$, 473,731 tests). All statistical analyses described here were performed using the MoBa 1 dataset. More information regarding these CpGs can be found in Additional file 1: Tables S1 and S2.

\section{GA prediction by ultrasound and LMP estimates}

To facilitate prediction of GA, we used Lasso regression from the elastic net package "glmnet" [18] (see "Methods" for more details on how and which regression models were tested). The Lasso-based regression model was trained with several different constellations of the MoBa 1 dataset. To reduce biases from factors assumed to influence GA prediction, we first trained the regression model with the FDR and Bonferronisignificant CpGs from both LMP- and ultrasoundbased regression models discussed in the previous section. In addition, we trained Lasso-regression models with the complete MoBa 1 dataset comprising 1068 samples (newborns) and 473,731 CpGs. The complete MoBa 2 dataset, containing 685 samples and 473,731 CpGs, was subsequently used for prediction of GA with the trained Lasso regression models. The Lasso-based GA predictions for both LMP and ultrasound were respectively compared to the LMP- and ultrasound-based GA estimations obtained from the MoBa cohort study using MM-type robust regression. As can be seen from Table 3, the performance of the LMP-based models was notably inferior to that of the ultrasound-based models in terms of both model fit $\left(R^{2}\right)$ and standard error measured as days within a $95 \%$ prediction interval. All Lasso models were comparable in performance but those trained with the complete MoBa 1 dataset retained substantially fewer CpGs than the other models; therefore, our primary focus will be on these models. A visualization of the performance of the MoBa 1 trained model can be seen in Fig. 3. Information 
Table 1 Covariates used in the preliminary regression models-MoBa 1

\begin{tabular}{lll}
\hline Covariate & Occurrence/mean value & $\mathrm{N}$ \\
\hline $\begin{array}{l}\text { Child's sex, male } \\
\begin{array}{l}\text { Mean age of } \\
\text { mother at birth }\end{array}\end{array}$ & $53.2 \%$ & $568 / 1068$ \\
$\begin{array}{l}\text { Maternal smoking } \\
\text { during pregnancy }\end{array}$ & $14.6 \%$ & 1068 \\
$\begin{array}{l}\text { Caesarian section } \\
\text { Asthma at 3 years }\end{array}$ & $11.5 \%$ & $156 / 1068$ \\
$\begin{array}{l}\text { Ultrasound } \\
\text { estimated GA }\end{array}$ & $272.9 \% .6(95 \%$ Cl 279-280.3) & $123 / 1068$ \\
LMP estimated GA & $282.3(95 \%$ Cl 281.6-283.00) & 1048 \\
\hline
\end{tabular}

$\mathrm{Cl}$ confidence interval

regarding the CpGs used in the respective prediction models can be found in Table 4 and Additional file 1: Table S3.

\section{Previously identified GA-related CpGs and genes}

As mentioned, previous studies have identified a number of significant CpGs with respect to GA. These studies were carried out using different DNA methylation assays, such as the previous generation Illumina HumanMethylation27 platform [6], the CHARM 2.0 assay [7], and more recently the Illumina HumanMethylation450 platform [10]. The number of individuals used in these studies was considerably smaller than in the present, with the exception of the Aries (ALSPAC)-based study, which included up to 974 samples [10]. Nevertheless, a total of 260 CpGs (representing 183 unique genes) were reported from both the Schroeder et al. [6] and Aries [10] studies, which was considerably fewer than the number of $\mathrm{CpGs}$ identified in the present study (Table 4). Out of the total $3654 \mathrm{CpGs}$ found to be differentially methylated with respect to GA in the MoBa cohort, 223 CpGs (linked to 138 genes) out $260 \mathrm{CpGs}$ were found to overlap with those found by the previous two studies (130 genes overlapped

Table 2 Study population -MoBa 2

\begin{tabular}{lll}
\hline Covariate & Occurrence/mean value & $\mathrm{N}$ \\
\hline Child's sex, male & $56.1 \%$ & $384 / 685$ \\
$\begin{array}{l}\text { Mean age of } \\
\text { mother at birth }\end{array}$ & $30.0(95 \%$ Cl 29.7-30.3) & 685 \\
$\begin{array}{l}\text { Maternal smoking } \\
\text { during pregnancy }\end{array}$ & $10.2 \%$ & $70 / 685$ \\
Caesarian section & $13 \%$ & $89 / 685$ \\
Asthma at 3 years & $21.3 \%$ & $104 / 489$ \\
Ultrasound & $279.4(95 \%$ Cl 278.5-280.2) & 644 \\
estimated GA & $281.5(95 \%$ Cl 280.7-282.4) & 615 \\
\hline LMP estimated GA & & \\
\hline
\end{tabular}

Cl confidence interval with the 183 genes from the Schroeder et al. and Aries studies). However, out of the 223 overlapping CpGs found to be significantly differentially methylated with respect to GA in our study, only $26 \mathrm{CpGs}$ (associated with 17 unique genes) were found to overlap with the CpGs used in the ultrasound/LMP-based prediction models, with only ten CpGs (covering five genes) overlapping among these again (Additional file 1: Table S4). It should be noted that the Schroeder et al. study [6] was based on the Illumina HumanMethylation27 platform and one CpG (cg20337106 mapping to gene C6orf139) was not present amongst the CpGs mapped by the HumanMethylation 450 platform. See Tables 4 and 5 (as well as Additional file 1: Tables S4) for more details.

The Lee et al. study [8] reported three genes, NFIX, MSRB3, and RAPGEF2, associated with GA. Since that study used the Charm 2.0 platform [7], which is based on a different technology to the Illumina HumanMethylation450 BeadChip employed in the present study, we checked for the presence of CpG sites, mapped by the Illumina platform, linked to the respective genes reported. Twelve CpGs were found for the NFIX gene, four for MSRB3 gene, and one for the RAPGEF2 gene amongst the total of 12,604 unique Bonferroni significant CpGs for both ultrasound- and LMP-based regression models. Out of the 131 unique significant CpGs employed in both ultrasound- and LMP-based GA prediction models, no overlapping genes were found with the genes identified by the Lee et al. study. Hence, while the genes reported by Lee et al. were found to have differentially methylated CpGs in our study, CpGs associated with these genes were not used in either the ultrasound- or LMP-based GA prediction models.

The 96 CpGs used to train the ultrasound-based GA prediction model mapped to 64 unique genes, while the 58 CpGs used to train the LMP-based GA prediction model mapped to 43 unique genes. Twenty-two genes were found to overlap between the ultrasound- and LMP-based prediction models. See Additional file 1: Tables S5 for more details regarding overlapping genes. The low number of overlapping CpGs between our ultrasound and LMP GA prediction models as well as the above-mentioned studies is puzzling and indicates that the association between GA and DNA methylation may be, more generally, linked to genome-wide development and/or changes to white blood cell profiles, which vary in ratio between cord blood and peripheral blood [10]. Cord blood is also known to contain stem cells and an increased number of stem cells that change DNA methylation profiles during gestation would most likely correlate with GA [19]. We did, however, try to correct for putative cell type influences using both principal components [20] and the method described 


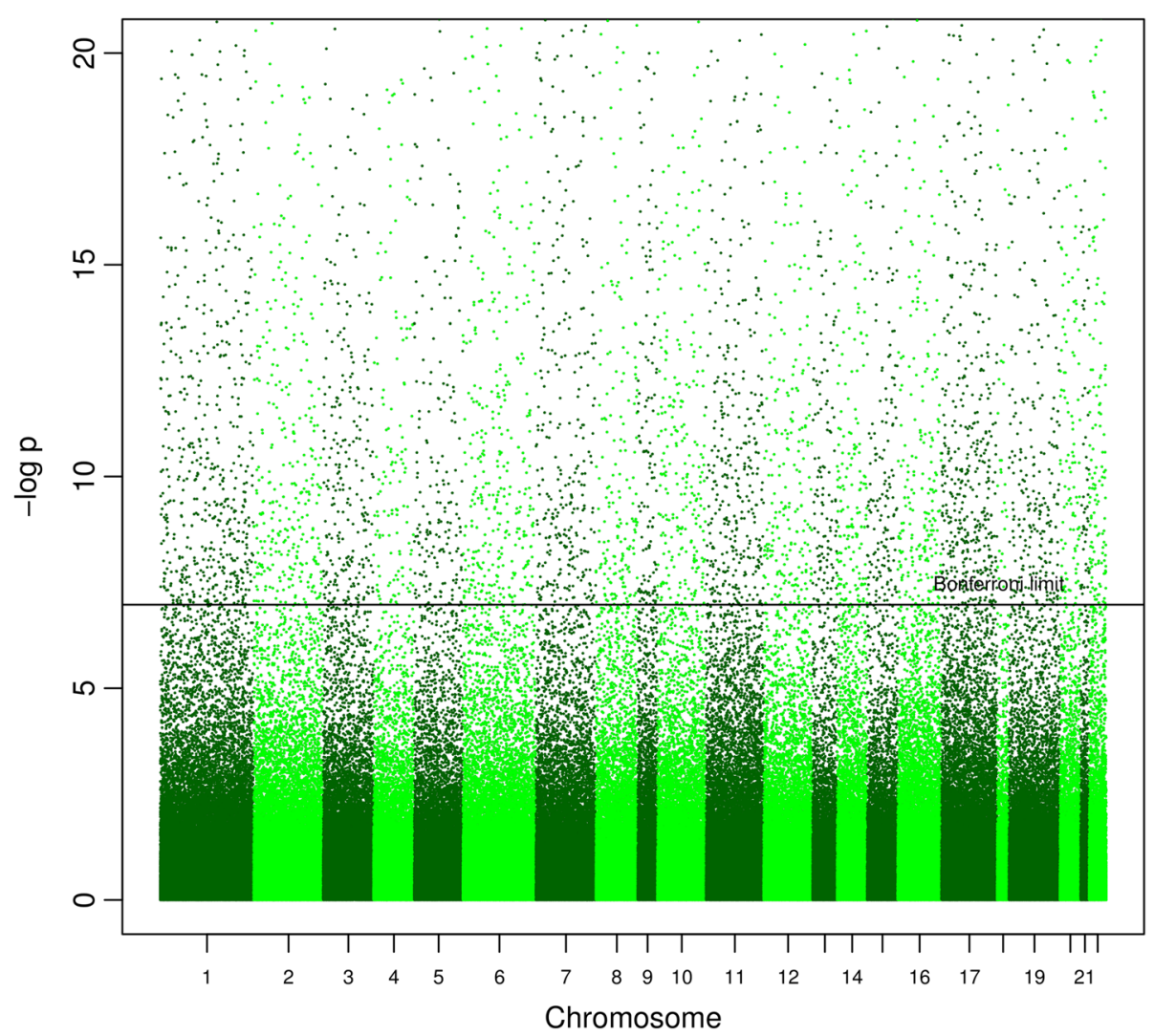

Fig. 1 A Manhattan plot of regression model-based estimates of 473,731 CpGs (response) with ultrasound estimated gestational age as the explanatory variable. The regression model was adjusted for selection bias, offspring sex, maternal smoking, caesarian section, and estimated cell-type differences

by Houseman [21] but with negligible effect with respect to the performance of the prediction models.

\section{Gene ontology}

The 22 genes found to overlap for both the ultrasound and LMP prediction models were examined with both DAVID (updated May 2016) [22, 23] and GOrilla (updated June 25, 2016) [24] databases in order to shed more light on the putative epigenetic influences responsible for the fairly accurate GA predictions observed in our study. However, FDR-significant associations were not identified with either DAVID or GOrilla $(\mathrm{q}>0.35)$ and since the results from DAVID did not add anything to those from GOrilla, we only present results from the latter. GOrilla identified "Nuclear matrix" (GO:0016363, genes "NCOR2", "DNMT3A", "HLCS", $p=2.32 \mathrm{E}-4$ ), "Chromatin" (GO:0000785, genes "NCOR2", "HGMA1", "DNMT3A", "HLCS", $p=6.19 \mathrm{E}-4$ ) (in the component ontology) "Positive regulation of cellular senescence" (GO:2000774, genes "HMGA1", "YPEL3", $p=2.49 \mathrm{E}-5$ ) "Positive regulation of cell aging" (GO:0090343, genes "HMGA1", "YPEL3", $p=3.48 \mathrm{E}-5)$, "Cellular response to ethanol" (GO:0071361, genes "DNMT3A", "ADCY7", $p=9.08 \mathrm{E}-5)$, "Negative regulation of gene silencing" (GO:0060969, genes "NCOR2", "HMGA1", $p=2.51 \mathrm{E}-4$ ), "Regulation of cellular senescence" (GO:2000772, genes "HMGA1", "YPEL3", $p=4.9 \mathrm{E}-4)$ and "Regulation of cell aging" (GO:0090342, genes "HMGA1", "YPEL3", $p=$ $8.05 \mathrm{E}-4)^{\prime \prime}$ (in the process ontology). While not FDRsignificant, it was of interest to note that several of the pathways identified were related to cell aging and cellular senescence.

\section{Gestational age and chronological age}

The prediction model for chronological age previously mentioned [12] was also based on a glmnet-trained model using 353 CpGs from the same Illumina HumanMethylation450 platform employed in the present study. These CpGs were compared to the ones used in both our ultrasound- and LMP-based GA prediction models. Surprisingly, we found that only one CpG (cg08965235 associated with the gene LTBP3) common to both ultrasound GA and chronological age prediction models (only cg08965235 and cg11299964, respectively, associated with 


\section{Volcano plot}

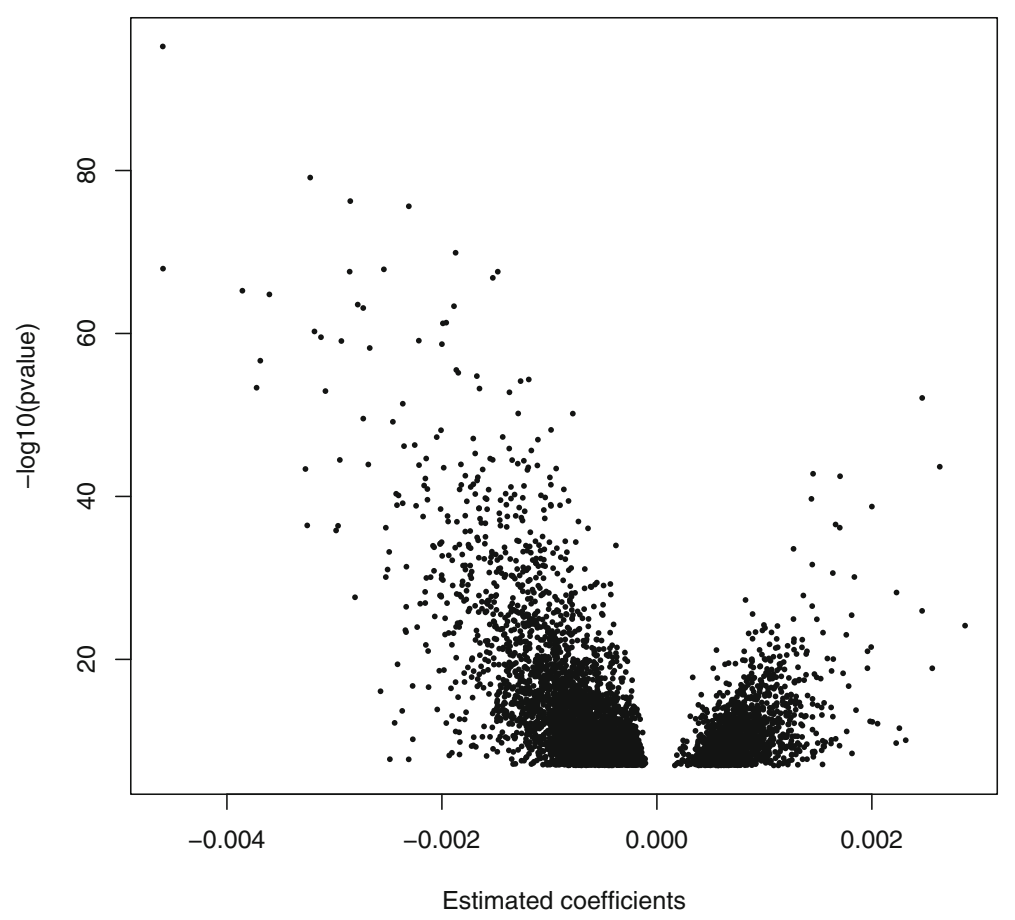

Fig. 2 A volcano plot based on the 5474 significant CpG coefficient estimates (horizontal axis), resulting from the ultrasound estimated GA-based regression models, plotted against the corresponding $p_{B}$ values (vertical axis). Negative coefficient estimates indicate decreased methylation, while positive estimates designate increased methylation

the genes LTBP3 and MAPKAP1 for the LMP GA prediction model). Since multiple CpGs mapped by the Illumina HumanMethylation450 BeadChip platform are linked to each gene, we also examined the number of overlapping genes and found one for the ultrasound-based prediction model, LTBP3 (two for LMP-based prediction model: LTBP3 and TOM1L1). The difference between GA- and chronological age-related DNA methylation has been examined in greater detail in the Aries cohort study, which included DNA methylation data from cord blood as well

Table 3 GA prediction results

\begin{tabular}{llll}
\hline & $\mathrm{R}^{2}$ & $\mathrm{SE}$ & Number of CpGs \\
\hline Ultrasound (Bonferroni adjusted) & 0.65 & 12.7 & 105 \\
LMP (Bonferroni adjusted) & 0.52 & 14.6 & 84 \\
Ultrasound (FDR adjusted) & 0.67 & 12.4 & 132 \\
LMP (FDR adjusted) & 0.53 & 14.5 & 107 \\
Ultrasound (MoBa 1) & 0.66 & 12.5 & 96 \\
LMP (MoBa 1) & 0.5 & 14.9 & 58
\end{tabular}

Boferroni/FDR refers to Lasso models trained with CpGs from the regression model adjusted for a set of covariates, including cell-type, as well as multiple testing. MoBa 1 models were trained using the complete MoBa 1 methylation data. The $\mathrm{R}^{2}$ column shows the goodness-of-fit statistics, based on MM-type robust regression, followed by standard error (SE) in \pm days ( $95 \%$ prediction interval) and the rightmost column designates the number of $\mathrm{CpGs}$ retained in each model as for ages 7, 15, and 17 years [10]. Furthermore, the substantially larger number of unique genes linked to the CpGs used by the respective prediction models (297, 64, and 43 genes for the chronological age and ultrasound and LMP GA prediction models, respectively) may suggest that different mechanisms linked to aging may be operating throughout life, something also mentioned in the Aries study [10]. The CpGs and genes that differed between the GA and chronological age prediction models can also be seen in Additional file 1: Tables S5.

\section{Practical implications}

The prediction model described here is based on DNA extracted from cord blood from newborns. Our GA predictor establishes a more assured link between GA and methylation, which may have important applications. For instance, since GA is related to many childhood health outcomes, being able to assess GA in circumstances where it is uncertain or unresolved could be critical for effective treatment. Furthermore, blood spots from newborns are routinely stored and can be useful to study in utero factors related to childhood disease. For such studies, GA can be determined by using methylation analyses and, thus, be adjusted for when a potential confounder. Although we 
Ultrasound

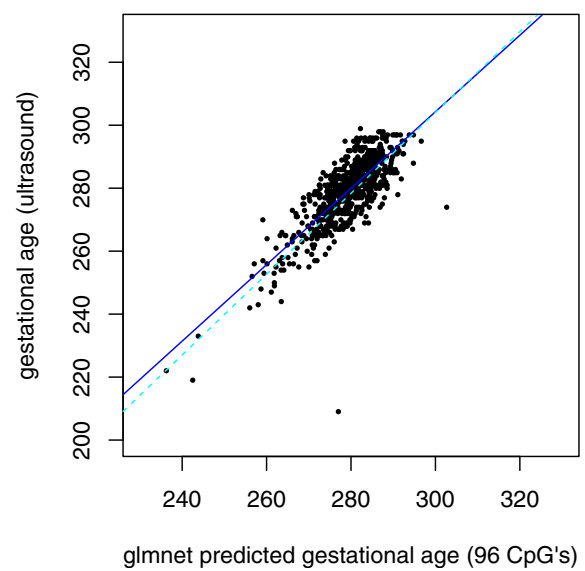

Last menstruation

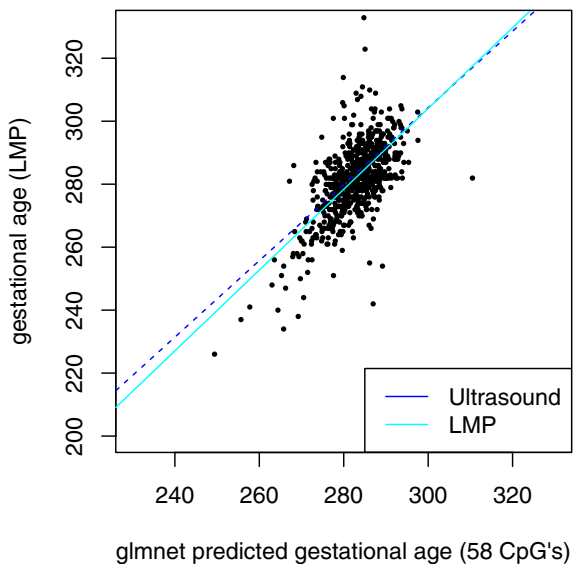

Fig. 3 Output from the regression models with ultrasound- and LMP-estimated gestational age (GA) as the response (vertical axis) and predicted GA as the explanatory variable (horizontal axis) in the left and right panels, respectively. The models, which were based on 96 (ultrasound) and 58 (LMP) CpGs, were trained using 1068 samples from MoBa 1. Prediction was carried out on 685 samples from MoBa 2. The dotted lines represent the adjacent regression estimates

used a class of models that are widely referred to as "prediction" models, for this purpose, we are "estimating" GA after birth rather than predicting it in advance. For true prediction before birth, one would need fetal blood while still in utero, which is difficult to obtain and extraction of which is associated with high risk. Using fetal cells in the maternal circulation, however, the method presented here might eventually enable actual prediction of GA and the due date in pregnant women.

\section{Conclusions}

We found that DNA methylation signatures at birth were strong predictors of GA. In addition, the prediction was more precise for ultrasound GA than for LMP GA. This is not unexpected, however, since ultrasound is generally regarded as a more reliable way to estimate GA amongst practitioners. Genes associated with chronological age do not appear to be strongly linked to genes associated with

Table 4 Number of CpGs associated with the different prediction/regression models

\begin{tabular}{ll}
\hline Ultrasound CpGs $p_{B}<0.05$ (FDR q<0.05) & $5474(44,359)$ \\
LMP CpGs $p_{B}<0.05$ (FDR q<0.05) & $10,784(44,544)$ \\
Common (intersect) & 3654 \\
Total unique CpGs (union) & 12,604 \\
Ultrasound predictor CpGs & 96 \\
LMP predictor CpGs & 58 \\
Overlapping predictor CpGs & 23 \\
Total unique predictor CpGs & 131 \\
Ultrasound unique predictor CpGs & 73 \\
LMP unique predictor CpGs & 35 \\
\hline
\end{tabular}

GA, suggesting that different epigenetic mechanisms are at work during different stages of life.

\section{Methods \\ Study population \\ MoBa cohort}

For this analysis, we included $1068+685$ newborns sampled at different occasions from the Norwegian Mother and Child Cohort Study (MoBa), which has previously been described in detail $[11,14,15]$ (Tables 1 and 2). The data collection in MoBa is approved by the Norwegian Data Inspectorate and the Institutional Review Board of the National Institute of Environmental Health Sciences, National Institute of Health, USA. The current study was also approved by the Regional Committee for Medical and Health Research Ethics of South East Norway.

\section{Variables}

GA estimates at birth were collected from the Medical Birth Registry of Norway, for which it is mandatory for health professionals at birth clinics to report birth outcomes. The birth registry provides two estimates for pregnancy length (GA), one based on ultrasound measurements

Table 5 Overlapping CpGs with previous studies

\begin{tabular}{lc}
\hline Total unique ultrasound and LMP CpGs $p_{B}<0.05$ & 12,604 \\
Significant CpGs in Schroeder/Aries studies & 260 \\
Overlapping ultrasound and LMP CpGs with Schroeder and & 223 \\
Aries studies & 131 \\
Unique predictor CpGs from both ultrasound/LMP models & 26 \\
Overlapping predictor CpGs with Schroeder and Aries & \\
\hline The number of CpGs associated with GA in our study found to overlap with \\
CpGs in other studies on GA
\end{tabular}


from around week 18 of pregnancy and one based on the maternally reported LMP. The birth registry also provided data on maternal age. Information on maternal education was obtained from the MoBa questionnaire for early pregnancies completed by the mother or based on data from the Medical Birth Registry of Norway (MBRN) [14, 15]. Additional details regarding the covariates particular to MoBa 1 in the present study can be found in Table 1 (Table 2 contains similar information for the MoBa 2 covariates).

\section{Pre-processing and quality control of methylation datasets MoBa 1}

The methylation data set used for training the prediction models consisted of 1204 Illumina HumanMethylation450 arrays based on cord blood DNA, each having 485,577 probes before quality control. Only singletons from unique mothers were included. Arrays not fulfilling the $5 \%$ detection $p$ value were removed together with all duplicates. Examination of plate effects revealed no bias; therefore, between-array normalization was not performed. However, within-array normalization was carried out using BMIQ from the wateRmelon package [25] to calibrate bias in type I and II probe technology. In addition to the Illumina control probes, we removed all probes on the $\mathrm{X}$ and $\mathrm{Y}$ chromosomes, resulting in total of 1068 arrays each consisting of 473,731 probes. For complete information and details regarding quality control of the Illumina HumanMethylation450 data used in the present study see $[11,26]$.

\section{MoBa 2}

This replication dataset consisted of the methylomes from 864 newborns each of which contained 485,577 probes. Only singletons from unique mothers were included in the dataset. All duplicates were removed and arrays not fulfilling the $5 \%$ detection $p$ value were excluded. Examination of the dataset revealed some mild-to-moderate plate effects; therefore, ComBat, utilizing empirical Bayes methodology, from the $\mathrm{R}$ package sva [27], was used for between-array normalization. Within-array normalization of type I and II probes was performed using BMIQ from the R package wateRmelon [25]. After exclusion of probes associated with the $X$ and $Y$ chromosomes, the Illumina control probes, and probes not found in the MoBa 1 dataset, the total number of probes was 473,731 for each of the 685 samples retained. Complete information regarding preparation of cord blood and quality control can be found in recent publications by Joubert et al. [28, 26].

\section{Statistical analysis}

MM-type robust linear regression [29] was first performed on the MoBa 1 newborn methylomes, with $\beta$ values
$(0 \leq \beta \leq 1)$ from each of the $473,731 \mathrm{CpG}$ sites as outcome variables. GA reported for the MoBa cohort (ultrasound/ LMP) was the explanatory variable. The regression models were adjusted for a set of covariates believed to be potential confounders. These included cell type composition estimates based on the Houseman procedure [21] (as computed from the minfi package with the Reinius dataset used as reference [30,31]), child's sex, maternal smoking, maternal age, study design (asthma diagnosed later at three years yes/no) and caesarian section (yes/no). Gestational age was based on (a) ultrasound measurements at around pregnancy week 18 and (b) reported LMP. Details regarding these covariates can be found in Tables 1 and 2 for both MoBa 1 and MoBa 2 datasets. We found $5474 \mathrm{CpGs}$ to be significant $\left(p_{B}<0.05\right)$ for ultrasound-estimated GA $(10,784$ CpGs for LMPestimated GA).

\section{Prediction models}

The 5474 and 10,784 CpG probes found to be significant $\left(p_{B}<0.05\right.$; as well as 44,359 and 44,544 FDR-significant $(\mathrm{q}<0.05)$ CpG probes) for ultrasound- and LMP-estimated GA from the regression models discussed in the previous section were added as predictors to "glmnet" elastic net models with $\alpha$ set to $0,0.5$, and 1 . The most appropriate $\alpha$ (i.e., type of regression method) was found using leaveone-out cross-validation. Lasso-type regression $(\alpha=0)$ resulted consistently in lower mean-squared error and was therefore our method of choice for the GA prediction models. The models based on $\alpha$ values of 0.5 and 1 where therefore not pursued further. Estimated Lasso penalties (also found using leave-one-out cross-validation) of 1 standard error above the minimum $\left(\lambda_{1 \mathrm{se}}\right)$, as suggested by Breiman [32], were preferred to minimum Lasso-penalties $\left(\lambda_{\min }\right)$ as the latter penalties resulted in models with only a marginally higher coefficient of determination $\left(R^{2}\right)$ than the former at the cost of retaining a substantially larger set of CpGs. To test the model's predictive abilities, we trained the Lasso model on all 1068 MoBa 1 samples with Bonferroni- and FDR-significant $\mathrm{CpGs}$ obtained from the regression model discussed in the previous section. In addition, the Lasso model was trained with the complete MoBa 1 dataset containing 473,731 CpGs and 1068 newborns. The corresponding CpGs from the 685 newborns in the MoBa 2 dataset were subsequently added to the trained Lasso models, which then returned estimated GAs for each newborn. All Lasso models were run with the same seed (1999). The predicted GAs for the 685 newborns from the Lasso model were regressed against the GA estimations reported by the MoBa cohort (response variable) using MM-type robust regression, the result of which can be observed in Fig. 3. The prediction model accuracy, in days, was reported 
as the $95 \%$ prediction interval based on the robust regression models (Table 3 ).

Since the "glmnet" model will not run with missing values, all missing GA estimations and probes were imputed using impute from the Hmisc R-package (median imputation) [33]. The CpGs used in the prediction model for chronological age are freely available as supplementary material from the study by Steve Horvath [12]. Further information regarding the CpGs used in the GA prediction models can be found in Additional file 1: Tables S3.

\section{Additional file}

Additional file 1: An Excel file containing information regarding CpGs and genes discussed in the present study. Table S1. All $p_{B}<0.05$ and $\mathrm{q}<$ 0.05 significant $\mathrm{CpGs}$ associated with ultrasound-estimated gestational age. Table S2. All $p_{B}<0.05$ and $\mathrm{q}<0.05$ significant $\mathrm{CpGs}$ associated with last menstrual period-estimated gestational age. Table S3. All unique CpGs used in both ultrasound and LMP prediction models. Table S4. CpGs reported by the Aries and Schroeder et al. studies overlapping with the CpGs used in the prediction models of the present study. Table S5. Information regarding genes associated with the CpGs from the different prediction models. (XLSX $8210 \mathrm{~kb})$

\section{Acknowledgments}

We are grateful to all families participating in the Norwegian Mother and Child Cohort Study. We also thank the reviewers for their suggestions and constructive remarks.

\section{Funding}

The Norwegian Mother and Child Cohort Study is supported by $\mathrm{NIH}(\mathrm{NIH} /$ NIEHS contract number N01-ES-75558, NIH/NINDS grant number 1 UO1 NS 047537-01 and grant number 2 UO1 NS 047537-06A1) and the Norwegian Research Council/FUGE (grant number 151918/S10). The work is also supported by the Norwegian Research Council/Human Biobanks and Health (grant number 221097 to W.N.) and in part by the Intramural Research Program of the NIH, NIEHS (ZIA ES049019) as well as the Norwegian Extra-Foundation for Health and Rehabilitation (grant number 2011.2.0218).

\section{Availability of data and material}

Access to the MoBa 1 and 2 DNA methylation data is available upon application to the Norwegian Institute of Public Health (NIPH). An application form in English can be found at the NIPH website (http://www.fhi.no/en/). Questions regarding the MoBa cohort can be directed to Wenche Nystad (Wenche.Nystad@fhi.no). An R package has been made that can be used to perform GA predictions. It is freely available under the open source GPL $(\geq 2)$ license at Zenodo (10.5281/zenodo.60498; https://zenodo.org/badge/latestdoi/ 23951/JonBohlin/predictGA).

\section{Authors' contributions}

$J B$ initiated the project, carried out interpretation of data and statistics, and wrote the manuscript and the software. SEH biological/physiological interpretation of results, wrote the manuscript. PM biological/physiological interpretation of results. SER statistical interpretation of results. HG: statistical help and guidance. MCM preparation of MoBa data and interpretation of results. CLP preparation of MoBa data and interpretation of results. CMP statistical interpretation of results. SJL interpretation of results and DNA methylation analysis. WN interpretation of results. All co-authors contributed to the writing of the manuscript. All authors read and approved the final manuscript.

\section{Competing interests}

The authors declare that they have no competing interests.

\section{Consent for publication}

Written informed consent was provided by all mothers participating in the study.

\section{Ethics approval and consent to participate}

The MoBa study was approved by the Regional Committee for Ethics in Medical Research, the Norwegian Data Inspectorate, and the Institutional Review Board of the National Institute of Environmental Health Sciences, USA. The experimental methods comply with the Helsinki Declaration.

\section{Author details}

${ }^{1}$ Norwegian Institute of Public Health, P.O. Box 44040456 Oslo, Norway. ${ }^{2}$ National Institute of Environmental Health Sciences, National Institutes of Health, Department of Health and Human Services, PO Box 12233MD A3-05, Research Triangle Park, Durham, NC 27709, USA.

Received: 11 April 2016 Accepted: 14 September 2016 Published online: 07 October 2016

\section{References}

1. Gjessing HK, Grottum P, Eik-Nes SH. A direct method for ultrasound prediction of day of delivery: a new, population-based approach. Ultrasound Obstet Gynecol. 2007;30(1):19-27.

2. Lynch CD, Zhang J. The research implications of the selection of a gestational age estimation method. Paediatr Perinat Epidemiol. 2007;21 Suppl 2:86-96.

3. Slama R, Khoshnood B, Kaminski M. How to control for gestational age in studies involving environmental effects on fetal growth. Environ Health Perspect. 2008;116(7):A284. author reply A284-A285.

4. Mongelli M, Wilcox M, Gardosi J. Estimating the date of confinement: ultrasonographic biometry versus certain menstrual dates. Am J Obstet Gynecol. 1996;174(1 Pt 1):278-81.

5. Bukowski R, Smith GC, Malone FD, Ball RH, Nyberg DA, Comstock CH, Hankins GD, Berkowitz RL, Gross SJ, Dugoff L, et al. Fetal growth in early pregnancy and risk of delivering low birth weight infant: prospective cohort study. BMJ. 2007:334(7598):836.

6. Schroeder JW, Conneely KN, Cubells JC, Kilaru V, Newport DJ, Knight BT, Stowe ZN, Brennan PA, Krushkal J, Tylavsky FA, et al. Neonatal DNA methylation patterns associate with gestational age. Epigenetics. 2011;6(12):1498-504.

7. Irizarry RA, Ladd-Acosta C, Carvalho B, Wu H, Brandenburg SA, Jeddeloh JA, Wen $B$, Feinberg AP. Comprehensive high-throughput arrays for relative methylation (CHARM). Genome Res. 2008;18(5):780-90.

8. Lee H, Jaffe AE, Feinberg Jl, Tryggvadottir R, Brown S, Montano C, Aryee MJ, Irizarry RA, Herbstman J, Witter FR, et al. DNA methylation shows genomewide association of NFIX, RAPGEF2 and MSRB3 with gestational age at birth. Int J Epidemiol. 2012;41(1):188-99.

9. Fraser A, Macdonald-Wallis C, Tilling K, Boyd A, Golding J, Davey Smith G, Henderson J, Macleod J, Molloy L, Ness A, et al. Cohort Profile: the Avon Longitudinal Study of Parents and Children: ALSPAC mothers cohort. Int J Epidemiol. 2013;42(1):97-110.

10. Simpkin AJ, Suderman M, Gaunt TR, Lyttleton O, McArdle WL, Ring SM, Tilling K, Davey Smith G, Relton CL. Longitudinal analysis of DNA methylation associated with birth weight and gestational age. Hum Mol Genet. 2015;24(13):3752-63.

11. Engel SM, Joubert BR, Wu MC, Olshan AF, Haberg SE, Ueland PM, Nystad W, Nilsen RM, Vollset SE, Peddada SD, et al. Neonatal genome-wide methylation patterns in relation to birth weight in the Norwegian Mother and Child Cohort. Am J Epidemiol. 2014;179(7):834-42.

12. Horvath S. DNA methylation age of human tissues and cell types. Genome Biol. 2013;14(10):R115.

13. Zbiec-Piekarska R, Spolnicka M, Kupiec T, Makowska Z, Spas A, Parys-Proszek A, Kucharczyk K, Ploski R, Branicki W. Examination of DNA methylation status of the ELOVL2 marker may be useful for human age prediction in forensic science. Forensic Sci Int Genet. 2015;14:161-7.

14. Magnus P, Irgens LM, Haug K, Nystad W, Skjaerven R, Stoltenberg C, MoBa SG. Cohort profile: the Norwegian Mother and Child Cohort Study (MoBa). Int J Epidemiol. 2006;35(5):1146-50.

15. Ronningen KS, Paltiel L, Meltzer HM, Nordhagen R, Lie KK, Hovengen R, Haugen M, Nystad W, Magnus P, Hoppin JA. The biobank of the Norwegian 
Mother and Child Cohort Study: a resource for the next 100 years. Eur J Epidemiol. 2006;21(8):619-25.

16. Sandoval J, Heyn H, Moran S, Serra-Musach J, Pujana MA, Bibikova M, Esteller M. Validation of a DNA methylation microarray for $450,000 \mathrm{CpG}$ sites in the human genome. Epigenetics. 2011;6(6):692-702.

17. Storey JD, Tibshirani R. Statistical significance for genomewide studies. Proc Natl Acad Sci U S A. 2003;100(16):9440-5.

18. Zou H, Hastie T. Regularization and variable selection via the elastic net. J R Statist Soc B. 2005;67(2):301-20.

19. Bocker MT, Hellwig I, Breiling A, Eckstein V, Ho AD, Lyko F. Genome-wide promoter DNA methylation dynamics of human hematopoietic progenitor cells during differentiation and aging. Blood. 2011;117(19):e182-9.

20. Chen W, Gao G, Nerella S, Hultman CM, Magnusson PK, Sullivan PF, Aberg $K A$, van den Oord EJ. MethylPCA: a toolkit to control for confounders in methylome-wide association studies. BMC Bioinformatics. 2013;14:74.

21. Houseman EA, Accomando WP, Koestler DC, Christensen BC, Marsit CJ, Nelson HH, Wiencke JK, Kelsey KT. DNA methylation arrays as surrogate measures of cell mixture distribution. BMC Bioinformatics. 2012;13:86.

22. da Huang W, Sherman BT, Lempicki RA. Bioinformatics enrichment tools: paths toward the comprehensive functional analysis of large gene lists. Nucleic Acids Res. 2009;37(1):1-13.

23. da Huang W, Sherman BT, Lempicki RA. Systematic and integrative analysis of large gene lists using DAVID bioinformatics resources. Nat Protoc. 2009; 4(1):44-57.

24. Eden E, Navon R, Steinfeld I, Lipson D, Yakhini Z. GOrilla: a tool for discovery and visualization of enriched $\mathrm{GO}$ terms in ranked gene lists. BMC Bioinformatics. 2009;10:48.

25. Pidsley R, Wong CCY, Volta M, Lunnon K, Mill J, Schalkwyk LC. A data-driven approach to preprocessing Illumina $450 \mathrm{~K}$ methylation array data. BMC Genomics. 2013;14:293.

26. Joubert BR, den Dekker HT, Felix JF, Bohlin J, Ligthart S, Beckett E, Tiemeier $H$, van Meurs JB, Uitterlinden AG, Hofman A, et al. Maternal plasma folate impacts differential DNA methylation in an epigenome-wide meta-analysis of newborns. Nat Commun. 2016;7:10577.

27. Leek JT, Johnson WE, Parker HS, Jaffe AE, Storey JD. The sva package for removing batch effects and other unwanted variation in high-throughput experiments. Bioinformatics. 2012;28(6):882-3.

28. Joubert BR, Felix JF, Yousefi P, Bakulski KM, Just AC, Breton C, Reese SE, Markunas CA, Richmond RC, Xu CJ, et al. DNA Methylation in newborns and maternal smoking in pregnancy: genome-wide consortium meta-analysis. Am J Hum Genet. 2016;98(4):680-96.

29. Yohai V, Stahel W, Zamar R. A procedure for robust estimation and inference in linear regression. In: Santosa F, editor. The IMA volumes in mathematics and its applications, vol. 34. New York: Springer; 1991. p. 365-74.

30. Aryee MJ, Jaffe AE, Corrada-Bravo H, Ladd-Acosta C, Feinberg AP, Hansen KD, Irizarry RA. Minfi: a flexible and comprehensive Bioconductor package for the analysis of Infinium DNA methylation microarrays. Bioinformatics. 2014:30(10):1363-9.

31. Reinius LE, Acevedo N, Joerink M, Pershagen G, Dahlen SE, Greco D, Soderhall C, Scheynius A, Kere J. Differential DNA methylation in purified human blood cells: implications for cell lineage and studies on disease susceptibility. PLoS One. 2012;7(7):e41361.

32. Breiman L, Friedman J, Olshen R, Stone C. Classification and Regression Trees. New York: Wadsworth; 1984.

33. Harrell Jr FE. Hmisc: harrell miscellaneous. R package version. 2008;3:4-4 http://crantastic.org/packages/Hmisc.

\section{Submit your next manuscript to BioMed Central and we will help you at every step:}

- We accept pre-submission inquiries

- Our selector tool helps you to find the most relevant journal

- We provide round the clock customer support

- Convenient online submission

- Thorough peer review

- Inclusion in PubMed and all major indexing services

- Maximum visibility for your research

Submit your manuscript at www.biomedcentral.com/submit
Biomed Central 